\title{
FAST ERROR-FREE ALGORITHMS FOR POLYNOMIAL MATRIX COMPUTATIONS
}

\author{
John S. Baras, David C. MacEnany and Robert L. Munach \\ Systems Research Center \\ University of Maryland \\ College Park, MD 20742
}

Abstract

Matrices of polynomials over rings and fields provide a unifying framework for many control system design problems. These include dynamic compensator desion, infinite dimensional systems, controllers for nonlinear systems, and even controllers for discrete event systems. An important obstacle for utilizing these powerful mathematical tools in practical applications ha been the non-availability of accurate and efficient algorithm to carry through the precise error-free computations required by these algebraic methods. In this paper we develop highly efficient, error-free algorithms, for most of the important computations needed in linear systems over fields or rings. We putations needed in inear systems over fields or rings. We critical in designing such algorithms.

\section{Introduction}

The theory of polynomial matrices $[9,22,24]$ plays a key role in the frequency-domain approach to the synthesis of multiple input multiple output control and communication systems $[14,25,26]$. Examples include coprime factorizations of transer function matrices, canonical realizations obtained from matrix fraction descriptions, design of feedback compensators and convolutional coders, and the analysis of quantization effect in linear systems. Typically, such problems abstract in a natural way to the need to solve systems of generalized Diophantine equations, e.g., the so-called Bezout equation $[7,16,20,23]$ These and other problems involving polynomial matrices require efficient polynomial matrix triangularization procedures [17], a result which is not surprising given the importance of matrix triangularization techniques in numerical linear alge bra. There, matrices with entries from a field can be triangularized using some form of Gaussian elimination. However, polynomial matrices have entries from a polynomial ring, an algebraic object for which Gaussian elimination is not de-
fined. For matrices with entries from a polynomial ring which fined. For matrices with entries from a polynomial ring which ory applications-triangularization is accomplished instead by what is naturally referred to as Euclidean elimination. Un fortunately, the numerical stability and sensitivity issues of Euclidean elimination are not well understood and in practice floating-point arithmetic has yielded poor results. At present a reliable numerical algorithm for the triangularization of polynomial matrices does not exist.

This paper presents algorithms for polynomial matrix triangularization which entirely circumvent the numerical senstivity issues of floating-point methods through the use of exact symbolic methods from computer algebra $[6,15,21]$. Often one encounters the comment that since in practical problems the numerical coefficients are rarely known very precisely, errorfree methods are an unecessary form of computational overkill. This is a misconception. The accuracy to which we know the coefficients is not the issue. The real issue is to what extent we can perform the required computations within the accuracy of the model data. Existing floating-point methods are poor highly sensitive and often lead to large errors, essentially since they suffer from the same problems as computing zeroes of polynomials. The use of exact, error-free algorithms guarantees that all calculations are accurate to within the precision of the model data - the best that can be achieved. Furthermore, one can calculate with such algorithms the exact sensitivities involved and therefore judge appropriately the confidence one should place on the results. Previous computer algebra algorithms for polynomial matrix problems appearing in control systems have been reported in [12]. Their performance was very slow even on small size problems.
We place emphasis on efficient algorithms to compute exact Hermite forms of polynomial matrices. The triangular, or more correctly, trapezoidal Hermite form is defined for any matrix with entries from a principal ideal ring $[22,24]$. Such matrices arise in many practical problems in communications and control. Here we shall focus on matrices having entries which are polynomials with rational coefficients, although our results easily abstract to more general settings [1]. An important aspect of the exact triangularization of such matrices involves the choice of arithmetic. We consider the tradeoffs between rational and integer arithmetic and choose the latter. This choice leads us to consider algorithms for the division of polynomials over a unique factorization domain (UFD). The standard algorithm for this task is well-known $[4,5,8,19]$ and defined more generally for polynomials with coefficients from any commutative ring with identity. This algorithm is well-suited to the scalar problem of GCD computation of polynomials over UFDs since it avoids the computation of GCDs of the coefficients. In the context of polynomial matrix triangularization however, it becomes unavoidable to exploit the richer structure of the coefficient ring: the fact that GCDs are defined on a UFD. As a result we present an alternative to the standard algorithm specialized to polynomials over UFDs but enjoying a certain optimality property which is crucial to the efficiency of matrix triangularization procedures.

We have implemented algorithms to compute exact Hermite forms of polynomial matrices in the MACSYMA and Mathematica computer algebra languages. We have also written a suite of auxiliary programs which call on these triangularization procedures in order to perform the more high-level tasks arising in the frequency-domain approach to control system synthesis. We conducted simulations with MACSYMA code running on Texas Instruments Explorer II and give performance results for the triangularization of polynomial matrices.

\section{Facts And Terminology of Polynomials} and Polynomial Matrices

In this section we use some standard terminology from modern algebra [11,13]; see also [1]. Denote by $Q[s]$ the ring of polynomials in the indeterminate ' $s$ ' with coefficients drawn from the field of rational numbers, $Q$. The subring $Z[s]$ of $Q[s]$ results when the polynomial coefficients are restricted to lie in $Z$, the ring of integers. A polynomial $a(s)$ in $Z[s]$ is called primitive if its coefficients are relatively prime in $Z$. For any $a(s)$ in $Z[s]$, there exists a non-zero scalar $c_{a}$ in $Z$, unique up to its sign, and a primitive polynomial $p_{a}(s)$ in $Z[s]$, such that $a(s)=c_{a} \cdot p_{a}(s)$. With slight imprecision $c_{a}$ is called the content of $a(s)$ and $p_{a}(s)$ its primitive (with respect to $c_{a}$ ). A collection of polynomials in $Z[s]$ having contents which are relatively prime we call relatively primitive.

Denote by $M[Q[s]$ the collection of $m \times n$ matrices with entries from $Q[s]$; we call $A(s)$ in $M[Q[s]]$ a polynomial matrix. Similarly, $M[Z[s]]$ will denote the subset of $M[Q[s]]$ when the entries are restricted to lie in $Z[s]$. We say that a row of a polynomial matrix $A(s)$ in $M[Z[s]$ is primitive if its polynomial entries are relatively primitive. We call $A(s)$ row (left) primitive, if every row is primitive. For any $A(s)$ in $M[Z[s]]$, there exists a diagonal matrix $C_{A}$ in $M[Z]$ and a row primitive matrix $P_{A}(s)$ in $M[Z[s]]$ such that $A(s)=C_{A} \cdot P_{A}(s)$; we call the pair $\left(C_{A}, P_{A}(s)\right)$ a left content-primitive factorization of $A(s)$. The diagonal elements of $C_{A}$ are the row contents of 
the respective rows of $A(s)$. By analogy with the scalar case, content-primitive factorization is obviously unique only up to the choice of the signs of the row contents.

For every $m \times n$ polynomial matrix $A(s)$ in $M[Q[s]]$ there exists a unimodular matrix $U(s)$ such that $U(s) A(s)=H_{A}(s)$ with $H_{A}(s)$ an upper triangular (trapezoidal) matrix satisfying the following conditions:

1. Each entry below the diagonal is identically zero;

2. Each nonzero diagonal entry has degree greater than the entries above it;

3. Each diagonal entry is monic.

We say that $H_{A}(s)$ is a column monic-Hermite form of $A(s)$. A column integral-Hermite form can be defined in terms of the column monic-Hermite form. Letting $H_{A}(s)$ denote a column monic-Hermite form for $A(s)$ in $M[Q[s]]$, multiply each row of $H_{A}(s)$ with the respectively smallest positive integer such that the matrix $H_{A}^{\prime}(s)$ so obtained is in $M[Z[s]]$. Clearly, $H_{A}^{\prime}(s)$ is row primitive and row equivalent to $A(s)$. Conversely, suppose that one is given $H_{A}^{\prime}(s)$ satisfying conditions (1) and (2) above which is row primitive and row equivalent to $A(s)$. Divide each row of $H_{A}^{\prime}(s)$ by the leading coefficient of the polynomial on the diagonal of the respective row and call the matrix so obtained $H_{A}(s)$. Then clearly there exists $U(s)$ unimodular such that $U(s) A(s)=H_{A}(s)$ and $H_{A}(s)$ is a monic-Hermite form of $A(s)$. This concept of column integral-Hermite form gives a triangular form in $M[Z[s]]$ for each matrix in $M[Q[s]]$. If $A(s)$ is nonsingular then it can be shown that its monicHermite form is unique and therefore its integral-Hermite form is also unique.

\section{Triangularizing Polynomial Matrices}

The upper triangularization of matrices with entries from a field using a sequence of non-singular (invertible) elementary row operations plays a key role in the application of the theory of vector spaces. Likewise, the upper triangularization of matrices with entries from a ring using a sequence of unimodular elementary row operations plays a key role in the application of the theory of vector modules. Computing triangular (trapezoidal) forms of matrices can be accomplished on any matrix module of the form $M[R]$ where $R$ is an integral domain, i.e., a commutative ring with identity having no zero divisors $[2,3,18]$. However, this cannot in general be accomplished using only unimodular (i.e., invertible) operations. Nevertheless, the transformation to an upper triangular form using unimodular elementary row operations can be performed quite straightforwardly - in theory at least-on any matrix with entries from the type of integral domain called a Euclidean ring, for instance on a matrix from $M[Q[s]]$. The key feature that Euclidean rings enjoy is the Euclidean division property which we state for $Q[s]$. Given polynomials $a(s), b(s)$ in $Q[s]$ with $\operatorname{deg} a(s) \leq \operatorname{deg} b(s)$ there exist two unique polynomials, the quotient $\bar{q}(s)$ and the remainder $r(s)$, such that $b(s)=q(s) a(s)+r(s)$ and $\operatorname{deg} r(s)<\operatorname{deg} a(s)$. The fact that the inequality on the degrees of $a(s)$ and $r(s)$ is strict allows one to introduce a zero into a polynomial matrix using elementary operations. The use of this process to introduce zeroes into polynomial matrices we call Euclidean elimination by analogy with Gaussian elimination.

\section{Integer vs Rational Arithmetic}

In a Euclidean elimination polynomials of the form $d(s)-$ $q(s) c(s)$ with $c, d, q$ in $Q[s]$ arise. To calculate the coeffcients of these forms one encounters the generic computation $\alpha+\beta \gamma$ with $\alpha, \beta, \gamma$ in $Q$. If these rationals are expressed as ratios of integers $\alpha=\frac{N^{\alpha}}{D^{\alpha}}, \beta=\frac{N^{\beta}}{D^{\beta}}, \gamma=\frac{N^{\gamma}}{D^{\gamma}}$, all reduced to lowest terms, then

$$
\alpha+\gamma \delta=\frac{N^{\alpha} D^{\beta} D^{\gamma}+N^{\beta} N^{\gamma} D^{\alpha}}{D^{\alpha} D^{\beta} D^{\gamma}} .
$$

This computation requires six integer multiplications, one integer addition and the calculation of a GCD. Although there are more efficient methods [18], it remains a fact that rational arithmetic is computationally expensive, due in large part to the need for GCD calculations. On the other hand, if it can be arranged so that $\alpha, \beta$ and $\gamma$ are all integers, then the same computation obviously requires only two integer multiplications, one integer addition and no GCD calculation. Thus, our goal is to carry out matrix triangularization on $M[Q[s]]$ using only integer arithmetic. Clearly, by multiplying each row of any $A(s)$ in $M[Q[s]]$ by a large enough integer, the denominators of every coefficient of every entry of $A(s)$ can be cancelled and such a diagonal operation is certainly unimodular in $M[Q[s]]$. Again, this computation can be arranged more efficiently but because it involves a fixed overhead, assume for convenience that $A(s)$ is given in $M[Z[s]]$.

Unfortunately, this creates new difficulties because Euclidean elimination is not defined for $M[Z[s]]$ since $Z[s]$ is not a Euclidean ring. For instance, it is easy to see that the remainder of two polynomials in $Q[s]$ with integer coefficients has, in general, rational coefficients; consider the remainder of $2 s$ after division by $3 s-1$. In other words, Euclidean division is not defined for $Z[s]$. However, $Z[s]$ is an instance of a polynomial ring with coefficients from a commutative ring with identity and for such a ring one has the pseudo-division lemma, a natural generalization of the Euclidean division lemma. Let $C$ denote a commutative ring with identity. Given $a(s)$ and $b(s)$ in $C[s]$ with $\operatorname{deg} a(s) \leq \operatorname{deg} b(s)$ there exist two polynomials, the pseudo-quotient $q(s)$ and the pseudo-remainder $r(s)$, such that $L b(s)=q(s) a(s)+r(s)$ and $\operatorname{deg} r(s)<\operatorname{deg} a(s)$ where the premultiplier $L=a_{0}^{\operatorname{deg} b-\operatorname{deg} a+1}$ with $a_{0}$ denoting the leading coefficient of $a(s)$. The pseudo-quotient and pseudoremainder are unique if $C$ is also an integral domain. The proof of the pseudo-division lemma yields a division procedure called pseudo-division which like Euclidean division enjoys the all-important strict degree reduction property; see [18] for the standard pseudo-division algorithm.

Let's consider an example in which we wish to pseudodivide $b(s)$ by $a(s)$ where,

$$
b(s)=s^{8}+s^{6}-3 s^{4}-3 s^{3}+8 s^{2}+2 s-5
$$

and

$$
a(s)=3 s^{6}+5 s^{4}-4 s^{2}-9 s+21 .
$$

Applying the standard pseudo-division algorithm one obtains,

$$
27 b(s)=\left(9 s^{2}-6\right) a(s)+\left(-15 s^{4}+3 s^{2}-9\right),
$$

i.e., $L=3^{8-6+1}=27, q(s)=9 s^{2}-6$ and $r(s)=-15 s^{4}+3 s^{2}-9$. This example appears in [18] as one step in the task of computing the GCD of $b(s)$ and $a(s)$. The next step is to divide out the content of $r(s)$ and then compute the GCD of $a(s)$ and $p_{r}(s)$ exploiting the fact that $\operatorname{gcd}(b(s), a(s))=\operatorname{gcd}\left(a(s), p_{r}(s)\right)$. The purpose of this content removal is to keep the size of the coefficients small for purposes of efficiency in succeeding calculations. However, consider the above computation in the context of a matrix triangularization-a $2 \times 2$ example will suffice:

$$
\left(\begin{array}{cc}
1 & 0 \\
-q(s) & L
\end{array}\right)\left(\begin{array}{ll}
a(s) & c(s) \\
b(s) & d(s)
\end{array}\right)=\left(\begin{array}{cc}
a(s) & c(s) \\
r(s) & L d(s)-q(s) c(s)
\end{array}\right) .
$$

In this situation we see that we are not at liberty to blindly divide the entire second row by the content of $r(s)$ (or any integer for that matter) because it may introduce rational coefficients in the $(2,2)$ entry and thereby ruin our attempt to maintain integer arithmetic. However, note that another solution to the above pseudo-division example is,

$$
9 b(s)=\left(3 s^{2}-2\right) a(s)+\left(-5 s^{4}+s^{2}-3\right),
$$

i.e., $L=27$ is not necessarily the smallest premultiplier for which a "pseudo-quotient" and "pseudo-remainder" exist. Obviously, in the matrix case, " $L=9$ " yields better results than $L=27$ since it yields smaller coefficients in the second row. Of course in this example the difference is negligible, however, if the size of the leading coefficient of $a(s)$ is large, the difference in computational burden can be quite substantial. Moreover as we shall see below, keeping the size of all coefficients as small as possible is a primary goal. 
5. Pseudo-division for Polynomials over a UFD It is apparent that there are smaller (and larger) premultipliers, $L$, than the one defined in the pseudo-division lemma. Now the pseudo-division lemma is the best that one can do in general for polynomials over a commutative ring with identity. But be aware that the concept of 'smaller' referred to in the pseudo-division example is inherited from the fact that $Z$ is also a unique factorization domain (UFD). Recall, a UFD is an integral domain which admits prime factorizations. Let $U$ denote a UFD. One can think of $u$ in $U$ as being "smaller" than $u^{\prime}$ in $U$ if $u$ is a divisor of $u^{\prime}$. For the problem of pseudodivision of polynomials $a(s), b(s)$ in $U[s]$, what we seek is the smallest premultiplier $L_{*}$ in $U$ such that if there exist $L$ in $U$ and $q, r$ in $U[s]$ satisfying,

$$
L b(s)=q(s) a(s)+r(s) \text { and } \operatorname{deg} r(s)<\operatorname{deg} a(s),
$$

then $L_{*}$ divides $L$ and $q_{*}(s), r_{*}(s)$ in $U[s]$ exist such that,

$$
L_{*} b(s)=q_{*}(s) a(s)+r_{*}(s) \text { and } \operatorname{deg} r_{*}(s)<\operatorname{deg} a(s) .
$$

The algorithm given next computes this $L_{*}, q_{*}(s)$ and $r_{*}(s)$ and is a distinct improvement over the pseudo-division lemma given in [18] for our purposes in that it computes with smaller numbers. It does so by exploiting the richer structure of polynomial rings with coefficients from a UFD but at the cost of both generality and GCD calculations. However, in the matrix problems we consider this cost is unavoidable.

Algorithm M - Pseudo-division of Polynomials over a UFD Given two nonzero polynomials $b(s)=b_{0} s^{n}+b_{1} s^{n-1}+\cdots+b_{n}$ and $a(s)=a_{0} s^{m}+a_{1} s^{m-1}+\ldots+a_{m}$ in $U[s]$ with $m \leq n$, this algorithm computes the smallest $L_{*}$, pseudo-quotient $q_{*}(s)$, and pseudo-remainder $r_{*}(s)$ as discussed above. It computes $L_{*}$, $q_{*}(s)$, and $r_{*}(s)$ directly by computing GCD's on the fly. This involves "smaller" numbers than first using the pseudo-division algorithm [18] and then computing GCDs. Bigger numbers cost more in GCD calculations and given the size of the in tegers encountered in polynomial matrix computations, e.g. easily greater than 1000 digits, this algorithm can save a substantial amount of time. For simpler notation we drop the 'asterisk' subscript in the algorithm's definition.

BEGIN:

$$
\begin{aligned}
& m n m \leftarrow \min (m, n-m) \\
& g \leftarrow G C D\left(b_{0}, a_{0}\right) \\
& l \leftarrow a_{0} / g \\
& L \leftarrow l \\
& b_{0} \leftarrow b_{0} / g \\
& \text { For } i=1 \text { thru } n-m \text { Do } \\
& \quad \text { For } j=i \text { thru } n-m \text { Do } \\
& \quad \text { EndDo } b_{j} \leftarrow b_{j} * l \\
& \quad \text { For } j=1 \text { thru } \min (m n m, n-m-i+1) \text { Do } \\
& \quad \text { EndDo } \\
& \quad \text { Endi-1 } \leftarrow b_{j+i-1}-a_{j} * b_{i} \\
& g \leftarrow G C D\left(b_{i}, a_{0}\right) \\
& \quad l \leftarrow a_{0} / g \\
& L \leftarrow L * l \\
& \quad b_{i} \leftarrow b_{i} / g \\
& \text { EndDo }
\end{aligned}
$$

END

The algorithm terminates with the first $n-m+1$ coefficients of $b(s)$ overwritten according to $\left\{b_{0}, b_{1}, \ldots, b_{n-m}\right\} \leftarrow$ $\left\{q_{0}, q_{1}, \ldots, q_{n-m}\right\}$ and the remaining coefficients over written according to $\left\{b_{n-m+1}, \ldots, b_{n}\right\} \leftarrow\left\{r_{0}, \ldots, r_{m-1}\right\}$.

$$
\begin{aligned}
& \text { Algorithm } \ddot{M}^{\prime}-\text { Proof of Correctness } \\
& \text { Alo }
\end{aligned}
$$

The informal language description of Algorithm $M$ basically implements the following recursion for $k=0,1, \ldots, n-m$,

$$
\begin{aligned}
b^{(-1)}(s) & =b(s) ; \\
g_{k} & =\operatorname{gcd}\left(a_{0}, b_{0}^{(k-1)}\right) ; \\
l_{k} & =a_{0} / g_{k} ; \\
p_{k} & =b_{0}^{(k-1)} / g_{k} ; \\
b^{(k)}(s) & =l_{k} b^{(k-1)}(s)-p_{k} a(s) .
\end{aligned}
$$

Observe that $\operatorname{deg} b^{(k)}(s)<\operatorname{deg} b^{(k-1)}(s)$ for $k=0,1, \ldots, n-m$ because $l_{k} b_{0}^{(k-1)}=p_{k} a_{0}$ (where $b_{0}^{(k)}$ of course denotes the leading coefficient of $\left.b^{(k)}\right)$. Hence, $\operatorname{deg} b^{(n-m)}(s)<\operatorname{deg} a(s)$. From the algorithm's definition we see that $r_{*}(s)=b^{(n-m)}(s)$ and $L_{*}=\prod_{k=0}^{n-m} l_{k}$. Solving the recursion above we obtain

$$
b^{(n-m)}(s)=L_{*} b(s)-q_{*}(s) a(s)
$$

where

$$
q_{*}(s)=\left(p_{0} l_{1} \cdots l_{n-m} s^{n-m}+\cdots+p_{n-m-1} l_{n-m} s+p_{n-m}\right) .
$$

The algorithm therefore yields,

$$
q_{*}(s)=\sum_{k=0}^{n-m}\left(\frac{L_{*}}{\prod_{i=0}^{k} l_{i}}\right) p_{k} s^{n-m-k},
$$

whence,

$$
L_{*} b(s)=q_{*}(s) a(s)+r_{*}(s) \quad \text { and } \quad \operatorname{deg} r_{*}(s)<\operatorname{deg} a(s) \text {, }
$$

with $L_{*}$ in $U$ and $q_{*}(s), r_{*}(s)$ in $U[s]$. Thus the algorithm indeed computes a valid solution; next we show that it is optimal. Suppose there exist another $L$ in $U$ and $q(s), r(s)$ in $U[s]$ such that,

$$
L b(s)=q(s) a(s)+r(s) \quad \text { and } \quad \operatorname{deg} r(s)<\operatorname{deg} a(s) .
$$

Then by commutativity $L_{*} L b(s)=L L_{*} b(s)$ implies,

$$
\left(L_{q_{*}}(s)-L_{*} q(s)\right) a(s)=L_{*} r(s)-L r_{*}(s) .
$$

Since there are no divisors of zero in a UFD this gives,

$$
\operatorname{deg}\left(L q_{*}(s)-L_{*} q(s)\right)+\operatorname{deg} a(s)=\operatorname{deg}\left(L_{*} r(s)-L r_{*}(s)\right) .
$$

Since $\operatorname{deg}\left(L_{*} r(s)-L r_{*}(s)\right) \leq \max \left\{\operatorname{deg} r(s), \operatorname{deg} r_{*}(s)\right\}<$ $\operatorname{deg} a(s)$ it must be true that,

$$
\operatorname{deg}\left(L q_{*}(s)-L_{*} q(s)\right)=-\infty
$$

and therefore $L_{*} q(s)=L q_{*}(s)$. By equating coefficients we obtain,

$$
L\left(\frac{L_{*}}{\prod_{i=0}^{k} l_{i}}\right) p_{k}=L_{*} q_{k} \quad k=0,1, \ldots, n-m,
$$

so that,

$$
q_{k} \prod_{i=0}^{k} l_{i}=p_{k} L \quad k=0,1, \ldots, n-m
$$

For $k=0$ we get $l_{0} q_{0}=L p_{0}$ and therefore $l_{0} \mid L p_{0}$. However, from the definition of the algorithm $l_{0}$ and $p_{0}$ are relatively prime in $U$, or coprime, and so in fact $l_{0} \mid L$. For $k=1$ we get $l_{0} l_{1} q_{1}=L p_{1}$ and therefore $l_{1} \mid\left(\frac{L}{l_{0}}\right) p_{1}$. Again, by construction $l_{1}$ and $p_{1}$ are coprime and so $l_{3} \mid \frac{t}{t_{0}}$. In general we have $l_{k}$ and $p_{k}$ coprime and $l_{k} q_{k}=\left(\frac{L}{l_{0} \cdots l_{k-1}}\right) p_{k}$ so that for $k=n-m$ we obtain,

$$
l_{n-m} \mid \frac{L}{l_{0} \cdots l_{n-m-1}} .
$$

As a result $l_{0} \cdots l_{n-m} \mid L$ and therefore $L_{*} \mid L$.

\section{Pseudo-Euclidean Elimination}

QED

The introducton of a zero below the diagonal of a matrix $A(s)$ in $M[Z[s]$ can now be performed using Algorithm $M$. This procedure we shall call pseudo-Euclidean elimination (PSEE) for obvious reasons. Consider triangularizing the matrix:

$$
A(s)=\left(\begin{array}{ccc}
1 & s & s \\
45 s & -10 s-10 & 3 s^{2}+s+10 \\
7-5 s & 6 s^{2}-1 & 4 s^{2}-10
\end{array}\right) .
$$


Pseudo-Euclidean elimination yields a matrix with first column [7577325 00 0 $]^{\prime}$, second column [0 891450$]^{\prime}$ and last column,

$$
\left(\begin{array}{c}
-1351755 s^{3}+1373940 s^{2}-5102550 s-7152750 \\
-43605 s^{3}+77103 s^{2}-234189 s-35190 \\
p_{33}(s)
\end{array}\right),
$$

where $p_{33}(s)$ is given by,

$3706425 s^{4}-5202000 s^{3}+18532125 s^{2}+15671025 s+7152750$

This illustrates the main disadvantage of triangularization on $M[Q[s]]$ performed over $M[Z[s]]$ - the coefficient growth of the polynomials. As the number of rows and columns in the matrix increases, this coefficient growth continues unabated and begins to erode the advantage of using integer arithmetic. One approach to handle this new source of coefficient growth is to remove the content of the current row after each pseudoEuclidean division step. It is better to remove the row content as soon as possible in this way rather than waiting due to the cost of computing GCDs of large integers, nevertheless, we illustrate row content removal for the current example. Factoring the above matrix into a left content-primitive form $C_{A} H_{A}^{\prime}(s)$ yields,

$$
C_{A}=\left(\begin{array}{ccc}
765 & 0 & 0 \\
0 & 9 & 0 \\
0 & 0 & 65025
\end{array}\right)
$$

and $H_{A}^{\prime}(s)$ equal to,

$$
\left(\begin{array}{ccc}
9905 & 0 & -1767 s^{3}+1796 s^{2}-6670 s-9350 \\
0 & 9905 & -4845 s^{3}+8567 s^{2}-26021 s-3910 \\
0 & 0 & 57 s^{4}-80 s^{3}+285 s^{2}+241 s+110
\end{array}\right) .
$$

The superfluous left content of the matrix can therefore be discarded since this is equivalent to multiplying it by $C_{A}^{-1}$ thereby keeping the coeffcient size to a minimum. We emphasize that $C_{A}$ is unimodular with respect to $M[Q[s]]$ but not with respect to $M[Z[s]]$. We stress that up to the signs of the entries across the rows $H_{A}^{\prime}(s)$ is the same matrix which would have resulted had we employed row content removal after each pseudo-Euclidean division step and that this is the more efficient strategy. Note that the above polynomial matrix $H_{A}^{\prime}(s)$ is nonsingular and in column integral-Hermite form and that therefore the unique column monic-Hermite form of $A(s)$ is obtained directly from $H_{A}^{\prime}(s)$ as,

$$
H_{A}(s)=\left(\begin{array}{ccc}
1 & 0 & -\frac{1767 s^{s}}{9905}+\frac{1796 s^{2}}{9905}-\frac{1334 s}{1981}-\frac{1870}{1981} \\
0 & 1 & -\frac{969 s^{3}}{1981}+\frac{8567 s^{2}}{9905}-\frac{26021 s}{9905}-\frac{782}{1981} \\
0 & 0 & s^{4}-\frac{80 s^{3}}{57}+5 s^{2}+\frac{241 s}{37}+\frac{110}{57}
\end{array}\right) .
$$

We see that PSEE provides an efficient triangularization procedure for $M[Z[s]]$ but, strictly speaking, PSEE modified with content factorization is not a valid triangularization procedure for $M[Z[s]]$ because content removal is not a unimodular operation in $M[Z[s]]$. On the other hand, augmenting PSEE with content factorization is a unimodular operation for $M[Q[s]]$ and yields an efficient triangularization procedure for $M[Q[s]]$ by avoiding rational arithmetic while maintaining integers of the smallest possible magnitude throughout an elimination.

7. Algorithms to Triangularize Polynomial Matrices Algorithm T - Column-Oriented Triangularization of Polynomial Matrices

Given an $N \times N$ nonsingular matrix $A \in M[Z[s])$, this algorithm overwrites $A$ with a triangular form obtained by a sequence of unimodular, elementary row operations. It avoids rational arithmetic by using pseudo-division as defined in Algo rithm $M$ in order to achieve maximum computational efficiency with minimum coefficient growth. In addition, it further inhibits coefficient growth by factoring out the row content after each pseudo-Euclidean division step. This algorithm operates in a column oriented fashion by successively zeroing out the entries in each column below the diagonal. This is shown pictorially below.

$$
\left(\begin{array}{lll}
x & x & x \\
x & x & x \\
x & x & x
\end{array}\right) \rightarrow\left(\begin{array}{lll}
x & x & x \\
0 & x & x \\
0 & x & x
\end{array}\right) \rightarrow\left(\begin{array}{lll}
x & x & x \\
0 & x & x \\
0 & 0 & x
\end{array}\right) .
$$

Assume there exists a pre-defined function,

$$
\text { MinDegIndex }(A, k):=\arg \min \left\{\operatorname{deg} A_{1, k}, \ldots, \operatorname{deg} A_{N, k}\right\} \text {, }
$$

which returns the index of the row of $A$ whose $k^{\text {th }}$ entry is a non-zero polynomial of lowest degree among the rows $\{k, k+$ $1, \ldots, N\}$. If $A_{k, k}(s)=A_{k+1, k}(s)=A_{N, k}(s) \equiv 0$, then it returns $-\infty$, the degree of the zero polynomial

BEGIN:

For $\mathbf{k}=1$ thru N-1 Do

index $\leftarrow$ MinDegIndex $(A, k)$

If index $\neq-\infty$ Then

$A_{k, .} \leftrightarrow A_{\text {index,. }}$ (exchange rows $k$ and index)

For $n=k+1$ thru $N$ Do

(zero out all entries in column $k$ below $A_{k, k}$ )

EndlessLoop

num $\leftarrow$ pseudo - quotient $\left(A_{n, k}, A_{k, k}\right)$

denom $\leftarrow$ pseudo - remainder $\left(A_{n, k}, A_{k, k}\right)$

$A_{n, .} \leftarrow$ denom $* A_{n, .}-$ num $* A_{k}$

$A_{n,} \leftarrow A_{n,} / G C D\left\{\right.$ content $\left(A_{n, 1}\right), \ldots$, content $\left.\left(A_{n, N}\right)\right\}$

If $A_{n, k} \equiv 0$ then exit EndlessLoop

$A_{n, .} \leftrightarrow A_{k}$,

End EndlessLoop

EndDo

EndIf

EndDo

End

Algorithm P - Principal Minor-Oriented Triangularization of Polynomial Matrices

This algorithm is similar to the one above except it performs the zeroing process in a leading principal minor oriented fashion so that the algorithm consists of $N-1$ stages where the $k \times k$ leading principal submatrix is in a triangular form by the end of the $k^{\text {th }}$ stage. Furthermore, the algorithm employs an additional substage which reduces the degrees of the polynomial entries above the diagonal on the $f y$ using pseudo-division as in Algorithm M. The order in which the degrees are reduced is important and is based upon notions from [17] for triangularizing matrices in $M[Z]$. The order is shown pictorially below.

$$
\begin{aligned}
\left(\begin{array}{llll}
x & x & x & x \\
x & x & x & x \\
x & x & x & x \\
x & x & x & x
\end{array}\right) & \rightarrow\left(\begin{array}{llll}
x & 1 & x & x \\
0 & x & x & x \\
x & x & x & x \\
x & x & x & x
\end{array}\right) \\
\left(\begin{array}{llll}
x & 2 & 3 & x \\
0 & x & 1 & x \\
0 & 0 & x & x \\
x & x & x & x
\end{array}\right) & \rightarrow\left(\begin{array}{llll}
x & 4 & 5 & 6 \\
0 & x & 2 & 3 \\
0 & 0 & x & 1 \\
0 & 0 & 0 & x
\end{array}\right) .
\end{aligned}
$$

The output matrix is in column integral-Hermite form, not simply triangularized as in Algorithm $T$, but with the entries above the diagonal of degree less than the diagonal entry. Clearly, the column monic-Hermite form is easily obtained by left multiplication with the appropriate diagonal matrix of rational numbers, a unimodular matrix with respect to $M[Q[s]]$. 
BEGIN:

For $k=2$ thru $N$ Do

For $n=1$ thru $k-1$ Do

(triangularize the $k \times k^{\text {th }}$ leading principal minor)

If $\operatorname{deg} A_{n, n}>\operatorname{deg} A_{k, n}$ Then $A_{k, .} \leftrightarrow A_{n,}$.

EndlessLoop

num $\leftarrow$ pseudoquotient $\left(A_{k, n}, A_{n, n}\right)$

denom $\leftarrow$ pseudoremainder $\left(A_{k, n}, A_{n, n}\right)$

$A_{k, .} \leftarrow$ denom $* A_{k, .}-$ num $* A_{n,}$

$A_{k,} \leftarrow A_{k} / G C D\left\{\right.$ content $\left(A_{k, 1}\right), \ldots$, content $\left.\left(A_{k, N}\right)\right\}$

If $A_{k, n} \neq 0$ then $A_{k, .} \leftrightarrow A_{n, .}$ else Exit EndlessLoop

End EndlessLoop

EndDo

For $i=-1$ thru $-k+1$ step -1 Do

(reduce degs of abv diag polys in $k \times k^{\text {th }}$ minor)

For $j=i+1$ thru 0 Do

If $\operatorname{deg} A_{k+i, k+j} \geq \operatorname{deg} A_{k+j, k+j}$ Then

num $\leftarrow$ pseudoquotient $\left(A_{k+i, k+j}, A_{k k+j, k k+j}\right)$

denom $\leftarrow$ pseudoremainder $\left(A_{k+i, k+j}, A_{k k+j, k k+j}\right)$

$A_{k+i, .} \leftarrow$ denom $* A_{k+i, .}-n u m * A_{k+j}$

$A_{k+i, .} \leftarrow A_{k+i, .} /$ $G C D\left\{\operatorname{content}\left(A_{k+i, 1}\right), \ldots, \operatorname{content}\left(A_{k+i, N}\right)\right\}$

EndIf

EndDo

EndDo

EndDo

End

We close this section by noting that in both Algorithm $T$ and Algorithm $P$ each pseudo-Euclidean division step affect the entire row and the row content is removed after each division step. Alternatively, one could solve a scalar Bezout identity for each zero to be introduced using pseudo-division tech niques and then perform a single elementary row operation followed by a single row content removal. However, the single row content of the latter method will be much larger than any of the "elementary" row contents computed by Algorithm T or Algorithm $P$. This makes the alternative method much less attractive than at first glance in light of the fact that computing the many "small" row contents is more efficient than computing the single "large" row content.

\section{Simulation Results}

Simulations were performed to determine the average time required to triangularize a square polynomial matrix and the maximum coefficient length of the output matrix using both Algorithm $T$ and Algorithm $P$ (see attached graphs). The maximum coefficient length is the number of digits of the largest (in absolute value) coefficient appearing in any polynomial entry of the output matrix. Each matrix had polynomial entries with randomly generated integer coefficients uniform on $[-99,99]$. Runs were parameterized by the dimension of the matrix, which ranged from 2 to 16 and the maximum degree of its polynomial entries, chosen uniformly on [0, degreemax], as degreemax ranged from 1 to 6 .

These simulations were conducted on a Texas Intruments Explorer II with $16 \mathrm{mb}$ of physical memory and $128 \mathrm{mb}$ of virtual memory running at $40 \mathrm{MHz}$ using the MACSYMA version of our algorithms. The graphs represent the results of the simulations averaged over 5 runs. The results indicate that Algorithm $T$ was moderately faster than Algorithm $P$ in triangularizing matrices up to $9 \times 9$. At that point Algorithm $T$ was still faster for triangularizing matrices with lower degree polynomials but slower in the higher degree polynomials This can be attributed to the fact that Algorithm $P$ requires less memory during computations due to its substage which reduces the degrees of the polynomials above the diagonal on the fly. Therefore costly garbage collections, a technique of freeing dynamically allocated memory, are reduced.

It appears that both of these algorithms run close to exponential time. The slopes of the semi-log plots of the timings increase slightly with increasing polynomial degree. The maximum coefficient length was approximately the same for each algorithm and the coefficient growth appears to be subexponential with increasing matrix dimension. A $16 \times 16 \mathrm{ma}-$ trix with degree 6 polynomials is the largest that has been attempted with Algorithm $P$. It required 40 hours to triangularize with the resulting matrix having a maximum coefficient length of 2115 digits.

Although Algorithm $T$ was faster than Algorithm $P$ on the smaller matrices, it did not have the overhead of putting the matrix into a canonic form in the process; Algorithm $P$ transforms the input matrix into the canonic integral-Hermite form as described earlier. The output matrix of Algorithm $T$ therefore requires the application of an auxilliary algorithm to reduce the degree of the polynomial entries above the diagonal in order to put it in strict integral-Hermite form. Of course this is not necessary if one is only interested in rank information.

If one keeps in mind the fact that our simulation results were run on full, random matrices, which tend to yield worstcase performance, then these simulations indicate that our algorithms in their current state are ideally suited for problems in which $\max \{m, n\} \leq 9$. Such problems include many practical control system designs, textbook problems in a classroom/lab environment, and empirical error analyses involved in research for alternative approaches to the machine computation of triangular forms of polynomial matrices based on other arithmetics such as floating-point or residue arithmetic [10]. For larger problems, our code can be modified in various ways to yield approximate results in much less time while providing some degree of error control. For instance, after the integer coefficients have reached a certain prespecified maximum size, the triangularization can be interrupted momentarily and the matrix $A(s)$ in $M[Z[s]]$ at its current state of triangularization can be converted to an associated matrix $A^{\prime}(s)$ in $M[Q[s]\}$ by premultiplication with a diagonal matrix in $M[Q]$. The matrix $A^{\prime}(s)$ can then be "floated" to any desired decimal precision and then re-expressed as a matrix in $M[Q[s]]$ and finally converted back to $M[Z[s]]$ to continue the triangularization. An ad hoc technique such as this is certainly approximate but if done properly can yield better results than the ad hoc floatingpoint techniques currently used. Refinements of this idea for Algorithm P with error bounds and simulation results will be appear elsewhere. We also compared our Hermite algorithm to the built-in Hermite algorithm included with the Scratchpad II and Maple computer algebra packages. On a $5 \times 5$ example generated randomly as above our code ran over 100 times faster.

\section{Summary of Functions}

The following is a summary of the high-level auxiliary programs which we have to date implemented in MACSYMA and Mathematica. They perform most of the common, high-level tasks arising in the frequency-domain approach to control system synthesis.

- RightMatrixFraction $(H(s))$ - Computes a right matrix fraction description of the transfer function matrix $H(s)$, i.e., computes the matrices $N(s), D(s)$ such that $H(s)=N(s) D(s)^{-1}$. The LeftMatrixFraction description is analogously computed.

- Bezout $(N(s), D(s))$ - Finds the homogenous and particular solutions to the Bezout equation, i.e., finds polynomial matrices $X_{h}(s), Y_{h}(s), X_{p}(s), Y_{p}(s)$ such that $X_{h}(s) D(s)+Y_{h}(s) N(s)=0$ and $X_{p}(s) D(s)+$ $Y_{p}(s) N(s)=I$. Used for designing feedback compensators in the frequency domain.

- ColumnReduce $(D(s))$ - Column reduces the polynomial matrix $D(s)$, i.e., multiplies $D(s)$ by an appropriate unimodular matrix such that the matrix of leading coefficients of its entries is nonsingular. RowReduce is analogously computed.

- Controller $(H(s))$ - Finds a controller form realization of the transfer function matrix $H(s)$. Controllability, Observer and Observability realizations are analogously computed.

- Hermite $(N(s))$ - Finds the canonic column Hermite form of the polynomial matrix $N(s)$. 
- RightCoprime $(N(s), D(s))$ - Determines the greatest common right divisor of the polynomial matrices $N(s)$ and $D(s)$. If it is not unimodular, it is factored out of both matrices making them right coprime. Used for finding minimal realizations. LeftCoprime is analogously computed.

- Smith $(N(s))$ - Finds the Smith form of the polynomial matrix $N(s)$. This is a canonic, diagonal form of a polynomial matrix.

- SmithMcMillan $(H(s))$ - Finds the Smith-McMillan form of the rational transfer function matrix $H(s)$. This is a canonic, rational, diagonal form of a matrix whose entries are ratios of polynomials.

Acknowledgements: This research was supported in part by NSF grant NSF CDR-8803012 under the Engineering Research Centers Program, and AFOSR University Research Initiative grant 87-0073. David MacEnany was partially supported by an IBM Fellowship.

\section{0 .References}

1] Baras, J.S., D.C. MacEnany and R.L. Munach "Fast Error-Free Algorithms for Polynomial Matrix Computations" Report SRC TR 90-14, Systems Research Center, University of Maryland, College Park, MD

[2] Bareiss, E.H. "Computational Solutions of Matrix Problems over an Integral Domain" J. Inst. Maths Applics V10, 69-104, 1972

[3] Bareiss, E.H. "Sylvester's Identity and Multistep IntegerPreserving Gaussian Elimination" Math. Comp. V22, 565-578, 1968

[4] Brown, W.S. "On Euclid's Algorithm and the Computation of Polynomial Greatest Common Divisors" J. ACM V18 (4) 478-504 Oct 71

5] Brown, W.S. and J.F. Traub "On Euclid's Algorithm and the Theory of Subresultants" J. ACM V18 (4) 505-514 Oct 71

[6] Buchberger, B. \& G.E. Collins et al (eds.) Computer Algebra: Symbolic and Algebraic Computation Wein: Springer, 1982

[7] Chou, T.J. and G.E. Collins "Algorithms for the Solution of Systems of Linear Diophantine Equations" Siam. J. Comp. V11 (4) 687-708 Nov 82

[8] Collins, G.E. "Subresultants and Reduced Polynomial Remainder Sequences" J. ACM V14 (1) 128-142 Jan 67

[9] Gantmakher, F.R. Theory of Matrices New York: Chelsea, 1959

[10] Gregory, R.T. and E.V. Krishnamurthy Methods and Applications of Error-Free Computation Berlin: Springer, 1984

[11] Hartley, B. and T.O. Hawkes Rings, Modules and Linear Algebra London: Chapman and Hall, 1970

[12] Holmberg, U. "Some MACSYMA Functions for Analysis of Multivariable Linear Systems" Technical Report CODEN:LUTFD2/(TFRT-7333)/1-040/(1986), Department of Automatic Control, Lund Institute of Technology, October 1986

[13] Hungerford, T.W. Algebra Berlin: Springer, 1974

14] Kailath, T. Linear Systems Englewood Cliffs: PrenticeHall, 1980

[15] Kaltofen, E. \& S.M. Watt (eds.) Computers and Mathematics Berlin: Springer, 1989

[16] Kannan, R. "Solving Systems of Linear Equations over Polynomials" Report CMU-CS-83-165, Dept. of Comp. Sci., Carnegie-Mellon University, Pittsburgh, 1983

[17] Kannan, R. and A. Bachem "Polynomial Algorithms for Computing the Smith and Hermite Normal Forms of an Integer Matrix" Siam. J. Comp. V8 (4) 499-507 Nov 79

[18] Keng, H.L. Introduction to Number Theory Berlin: Springer, 1982

[19] Knuth, D.E. The Art of Computer Programming, V2 Reading, Mass: Addison Wesley,1981

[20] Krishnamurthy, E.V. Error-Free Polynomial Matrix Computations Berlin: Springer, 1985
[21] Lipson, J.D., Elements of Algebra and Algebraic Computing Reading: Addison-Wesley, 1981

[22] MacDuffee, C.C. The Theory of Matrices New York: Chelsea, 1950

[23] McClellan, M.T. "The Exact Solution of Systems of Linear Equations with Polynomial Coefficients" J. ACM V20 (4) 563-588 Oct 73

[24] Newman, M. Integral Matrices New York: Academic Press, 1972

[25] Vidyasagar, M., Control System Synthesis Cambridge: MIT Press, 1985

[26] Wolovich, W.A., Linear Multivariable Systems Berlin Springer, 1974

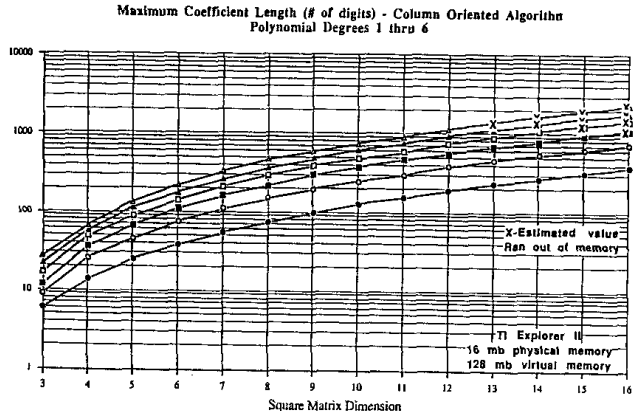

Maximum Coefficient Length (\# of digits) - Minor Oriente Polynomial Degrees 1 thru 6

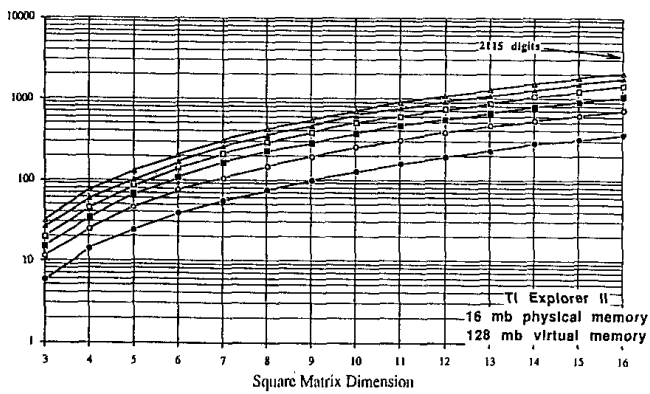

Tlme to Triangularke (sec) - Column Oriented Algorlthm
Polynomial Degrees I thru 6
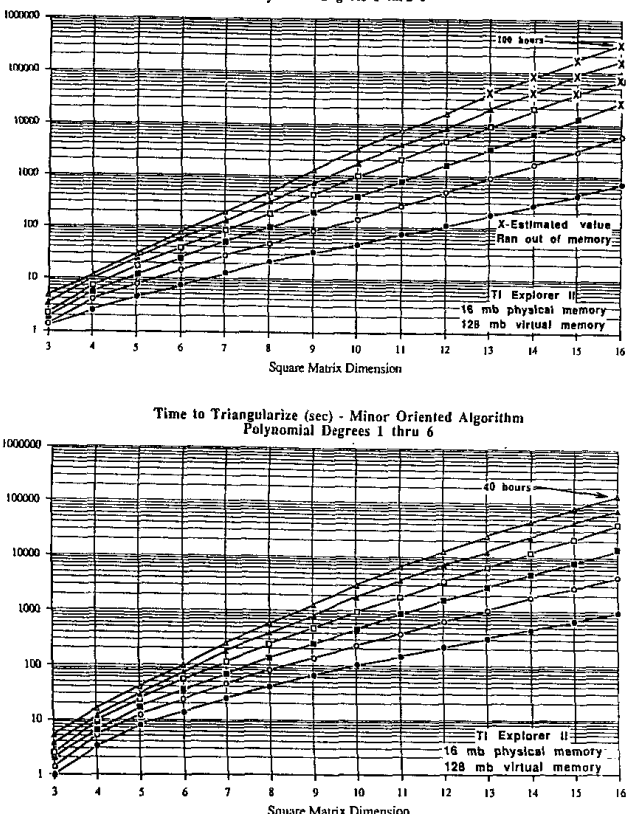